\title{
Fabrication and testing of corrugated waveguides for a collinear wakefield accelerator
}

\author{
A. Siy $\odot,{ }^{1,2,}{ }^{*}$ N. Behdad, ${ }^{1}$ J. Booske, ${ }^{1}$ M. Fedurin, ${ }^{3}$ W. Jansma $\odot,{ }^{2}$ K. Kusche $\odot,{ }^{3}$ S. Lee, ${ }^{2}$ \\ G. Mouravieff, ${ }^{4}$ A. Nassiri, ${ }^{2}$ S. Oliphant $\odot{ }^{4}$ S. Sorsher, ${ }^{2}$ K. Suthar, ${ }^{2}$ E. Trakhtenberg, ${ }^{2}$ \\ G. Waldschmidt, ${ }^{2}$ and A. Zholents $\oplus^{2, \dagger}$ \\ ${ }^{1}$ University of Wisconsin, Madison, Wisconsin 53715, USA \\ ${ }^{2}$ Advanced Photon Source, Argonne National Laboratory, Argonne, Illinois 60439, USA \\ ${ }^{3}$ Brookhaven National Laboratory, Upton, New York 11973 \\ ${ }^{4}$ Servometer, Cedar Grove, New Jersey 07009, USA
}

(Received 16 November 2021; accepted 3 February 2022; published 17 February 2022)

\begin{abstract}
Significant progress has been made at Argonne National Laboratory in the development of a compact wakefield accelerator based on a cylindrical corrugated waveguide with a 2-mm ID and fine corrugations on the wall. The fabrication process of 10-cm-long corrugated waveguide structures has been established and a high quality of the final product has been confirmed by precision metrology. Several samples have been tested using the electron beam at Brookhaven National Laboratory's Accelerator Test Facility. The frequency and group velocity of the fundamental monopole $\mathrm{TM}_{01}$ mode of sub-terahertz Čerenkov radiation produced by a 1.5-ps-long electron bunch propagating in the corrugated waveguide, as well as the frequencies of the dipole $\mathrm{HEM}_{11}$ and the quadrupole $\mathrm{HEM}_{21}$ modes have been measured and found to be in good agreement with the design values calculated using CST Microwave Studio. The energy modulation of a 5-ps-long electron bunch caused by the self-induced wakefield has also been measured and found to be in good agreement with the calculated values.
\end{abstract}

DOI: 10.1103/PhysRevAccelBeams.25.021302

\section{INTRODUCTION}

A high-charge drive electron bunch generates an electromagnetic field passing through a cylindrical corrugated waveguide (CWG) structure. This field, called the wakefield, is used to accelerate a low charge witness electron bunch, which propagates behind the drive bunch as proposed in [1-6]. The peak value of the wakefield is proportional to $Q / a^{2}$, where $Q$ is the drive bunch charge and $a$ is the radius of the CWG. Calculations show that a peak electric field of $\sim 100 \mathrm{MV} / \mathrm{m}$ can be obtained using a drive bunch with $Q \simeq$ $10 \mathrm{nC}$ and a CWG with $a=1 \mathrm{~mm}$ [7]. Fabrication of such a CWG is challenging because the depth and width of the corrugations are only on the order of a few hundreds of microns. After exploring several fabrication techniques, we chose the electroforming method and discuss this fabrication process in detail in Sec. II. In Sec. III we discuss the experimental results obtained during testing of the CWG samples with an electron beam and compare them with

\footnotetext{
*asiy@wisc.edu

azholents@anl.gov
}

Published by the American Physical Society under the terms of the Creative Commons Attribution 4.0 International license. Further distribution of this work must maintain attribution to the author(s) and the published article's title, journal citation, and DOI. calculations. Various CWG applications for accelerators and light sources have been actively studied in the past decade theoretically [8-10] and experimentally. The CWGs with a planar geometry have been successfully employed to cancel linear energy correlation in the electron bunch and thus to enhance the performance of free electron lasers [11-13]. Using the cylindrical CWG as a passive streaker for a time resolved diagnostic of the electron bunches was proposed in [14]. Using the cylindrical CWG for a subterahertz free electron laser was proposed in [15]. In Ref. [16] authors report fabrication and testing of a 50-mm long cylindrical CWG with a quasisinusoidal profile of corrugations. It was used to produce a narrow-band subterahertz radiation.

\section{FABRICATION OF THE CYLINDRICAL CORRUGATED WAVEGUIDE}

Physics requirements for the CWG described in [17] prompted selection of a CWG with the dimensions shown in Fig. 1. Fabrication of a CWG with these dimensions and a length of $0.5 \mathrm{~m}$ presents significant manufacturing challenges. To address this, we investigated many methods for making CWG structures, including die stamping, pressing, optical lithography, electrical discharge machining, laser ablation, micro-machining, and electroforming. A brief survey of these methods is reported below (see, also [18] for more details). 


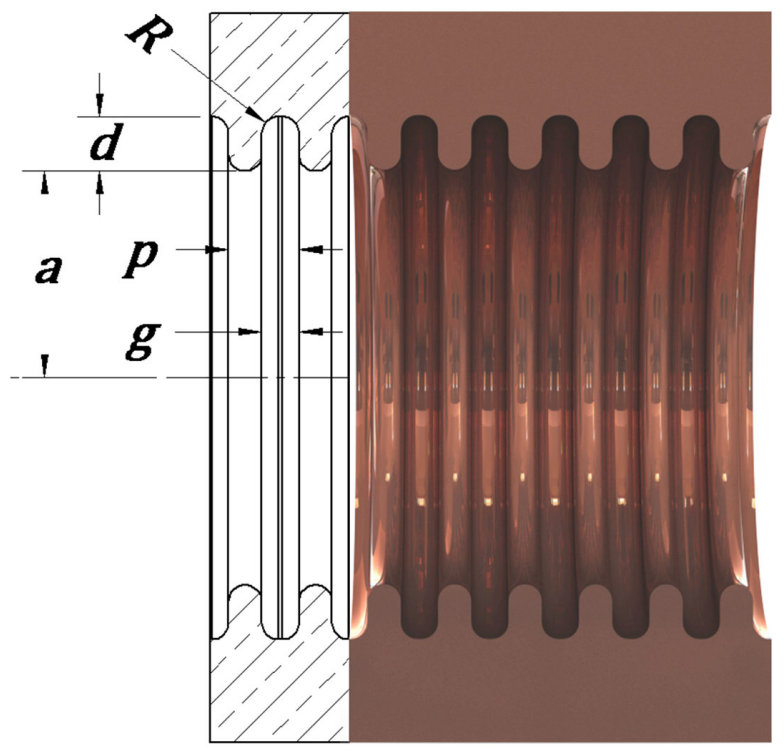

FIG. 1. Dimensions of the cylindrical corrugated waveguide: $\mathrm{a}=1 \mathrm{~mm}, \mathrm{p}$ (period) $=340 \mu \mathrm{m}, \mathrm{g}$ (gap) $=180 \mu \mathrm{m}, \mathrm{d}$ (depth) $=264 \mu \mathrm{m}, \mathrm{R}$ (corner radius) $=80 \mu \mathrm{m}$.

\section{A. Survey of fabrication techniques}

A small section of CWG was fabricated using each method to evaluate the method's cost and capability of producing the desired corrugation profile to better than $10 \mu \mathrm{m}$ accuracy. A Keyence VR3200 3D surface profiler was used to perform high-precision metrology of the fabricated structures as reported in [19]. The instrument works by projecting structured white light patterns on a sample that are received by a CMOS detector to produce a $3 \mathrm{D}$ surface profile with $<1 \mu \mathrm{m}$ vertical resolution and $1 \mu \mathrm{m}$ spatial resolution.

\section{Die stamping}

One corrugation period can be divided into two parts, a ridge and a trough, as shown in Fig. 2(a). The thickness of the ridges is $160 \mu \mathrm{m}$, and the thickness of the troughs is $180 \mu \mathrm{m}$. There is a total of 1472 corrugations per 0.5 -meter-long waveguide. Ridges and troughs can be manufactured individually as rings of copper $(\mathrm{Cu})$ foil and alternately combined in series to form a long waveguide. A solid structure can then be achieved by brazing. Two stamping dies were created for the ridges and troughs and used to stamp $\mathrm{Cu}$ foil with thicknesses of $160 \mu \mathrm{m}$ and $180 \mu \mathrm{m}$, respectively. It was found that the stamping method required precision cutting to maintain the inner dimensions along the circumference. Also, due to the large number of joints in the overall structure, quality control is very challenging with this method to ensure the proper alignment of each sequence of ridge and trough.

\section{Pressing}

This technique uses the plastic deformation principle to make a corrugated indentation pattern on a
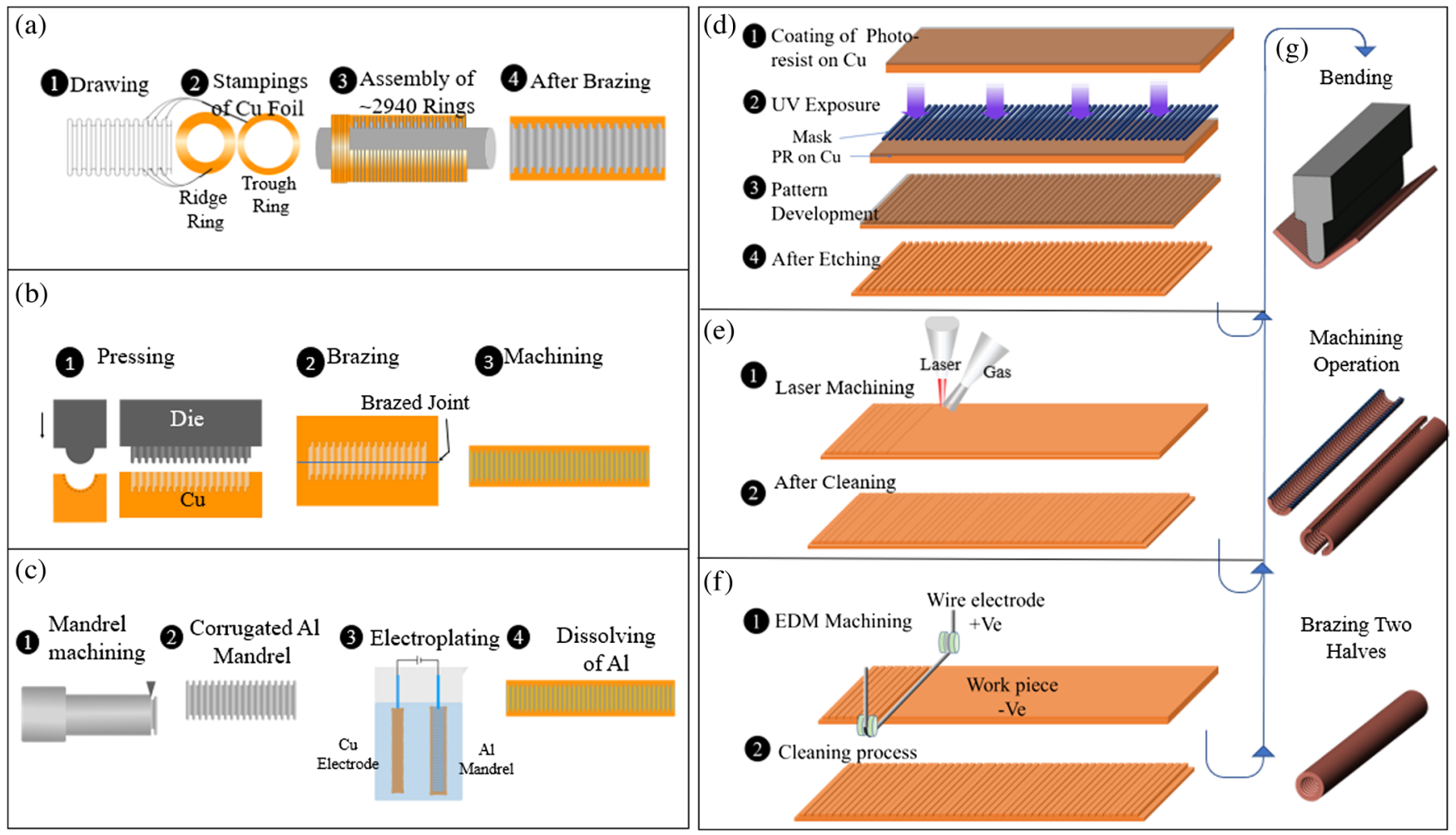

FIG. 2. Figures show various fabrication techniques that were investigated for production of the corrugated waveguide: (a) die stamping, (b) pressing, (c) electroforming, (d) optical lithography, (e) laser ablation, (f) electrical discharge machining. 
semicircular-shaped $\mathrm{Cu}$ form. A schematic is shown in Fig. 2(b). A semicircular die was prepared with an inverse pattern of the corrugations. The base diameter was kept equivalent to the inner diameter of the corrugated waveguide; thus upon deformation, the $\mathrm{Cu}$ conforms to the required shape. Two $\mathrm{Cu}$ pieces were machined, each with a 2-mm-diameter semicircular channel. The die is pressed against the $\mathrm{Cu}$ channel to make an impression in the circular surface. After iterative pressing and translating along the centerline of the 0.5 -meter-long $\mathrm{Cu}$ structure, the patterned sections will be joined by brazing. The pressing or embossing technique has merit since the initial base structure can be kept straight and aligned after the brazing process. After brazing, the whole part can be machined to achieve the required shape. The biggest challenge here is to align the two halves of corrugated waveguide along the length and manage the flow of melted brazing alloy in such a way that all corrugations are joined without spillover in the adjacent corrugation profiles. This challenge can be mitigated by including an appropriately sized capillary channel next to the corrugations and providing fiducials for an accurate alignment. It remains challenging to stop the flow of brazing alloy into the corrugations.

\section{Optical lithography}

In this technique, the corrugations were first etched on a planar surface via an optical lithography technique [refer to Figs. 2(d) and 2(g)]. Photoresist was coated on a flat $\mathrm{Cu}$ plate followed by exposure to UV light through a grating mask made with the corrugation dimensions. The exposed photoresist was removed using a chemical process followed by etching with a $\mathrm{FeCl}_{3}$ solution. One can see a modulation in patterning, which could have originated from crosslinking in the photoresist. The corrugation depth also fluctuated every $\sim 3 \mathrm{~mm}$. This can create unwanted amplitude and frequency modulation in the wakefield.

The flat plate was then cut in appropriate widths and the parts were bent into semicircular channels. These channels were joined by laser welding which was a labor-intensive process. The weld beads were hand-laid by a highly skilled craftsman using $125 \mu \mathrm{m}$-diameter $\mathrm{Cu}$ wire as the filler material. The workpiece motion was coordinated with the laser pulses and the operator added material while viewing the workpiece through a stereomicroscope. To test the method, several noncorrugated half-tubes were joined by laser welding. A 3D image of a test sample showed that the nominal width of the hand-laid weld bead was only $0.6 \mathrm{~mm}$. Results indicated overall good quality in the joints, although a few penetrations to the inside of the tube were evident.

Electron beam welding of the tubes was attempted by our colleagues at Jefferson National Lab, but it was determined to be problematic due to heating and expansion of the thin walls of the tube during welding caused by the high thermal conductivity of $\mathrm{Cu}$.

\section{Electrical discharge machining}

The planar structures with corrugations were fabricated using electrical discharge machining (EDM) Fig. 2(f) and followed the same method of fabrication as shown in Fig. 2(g). It also carried similar challenges of joining the two halves after bending.

\section{Laser ablation}

Similar to photolithography and EDM, the structures were fabricated using laser ablation machining (see Fig. 2e and followed the same method of fabrication afterward [see Fig. 2(g)]. This method carried similar challenges of joining the two halves after bending regarding alignment accuracy and brazing containment.

\section{B. Electroforming}

Electroforming or electroplating is a well-established technology (see [20] and references therein), and we found it to be the most successful method to produce the CWG. Figure 2(c) shows the main electroforming steps. In brief, an aluminum $(\mathrm{Al})$ mandrel is produced by micro-machining. After precision masking is applied, the mandrel is placed in a plating tank for several days where copper sulfate with proprietary additives is used for copper deposition. Afterwards, the mandrel is placed in a hot bath of $\mathrm{NaOH}$ to chemically dissolve the aluminum. The complete etching of aluminum leaves behind the plated structure as the final electroformed product. The corrugation dimensions reflect the manufacturing quality of the aluminum mandrel geometry. The surface roughness ( $\mathrm{Ra})$ of the electroformed components depend on the deposition thickness and the surface roughness of the $\mathrm{Al}$ mandrel. The CWG fabrication requires a $0.5-\mathrm{mm} \mathrm{Cu}$ deposition thickness. With this thickness we should be able to obtain $\mathrm{Ra} \simeq$ $700 \mathrm{~nm}$ using a hybrid electrochemical process [21], in which case the surface roughness of the Al mandrel will likely determine the surface roughness of the final product.

The maximum CWG length is limited by the ability to maintain straightness of the 2.53-mm-diameter $\mathrm{Al}$ mandrel during machining. Since making a 0.5 -meter-long waveguide as one piece is not feasible, we consider brazing five 98-mm-long sections, as shown in Fig. 3.

Metrology analysis of the CWG section mandrel shown in Fig. 4 indicates an excellent uniformity in the corrugation pattern and a good surface finish. The tooth's height and width are $252.7 \pm 1.5 \mu \mathrm{m}$ and $175.9 \pm 2.0 \mu \mathrm{m}$, correspondingly. The design values are $264 \mu \mathrm{m}$ and $160 \mu \mathrm{m}$. We estimate that these errors can lead to up to $4 \%$ uncertainty in the frequency of the fundamental mode of the wakefield.

The Al mandrels develop a sag during machining with the measured offsets in the center distributed anywhere from 40 to $100 \mu \mathrm{m}$ with a mean value of $65 \mu \mathrm{m}$ and a standard deviation of $18 \mu \mathrm{m}$. These offsets have been 

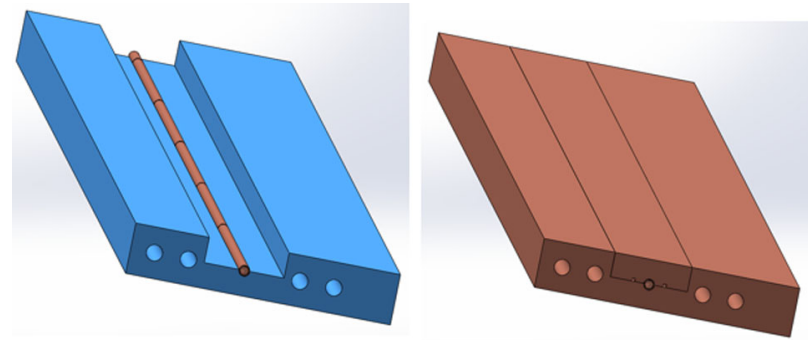

FIG. 3. Brazing assembly of the 0.5-meter-long waveguide.

reduced to below the acceptable tolerance of $4 \mu \mathrm{m}$ with a manual straightening using a fixture that is controlled by observation on an optical comparator with a modest resolution of approximately $2.5 \mu \mathrm{m}$.

Once electroforming of the mandrel is complete, the plated electroform is machined while still on the mandrel with reference to the masked surfaces. EDM is performed using a custom-made tungsten electrode to obtain the $2.9 \mathrm{~mm}$ outside diameter (OD) of the plated mandrels required by the design. An extra $\mathrm{Cu}$ layer was deposited during electroforming to accommodate possible plating depth irregularities and obtain a concentric OD with respect to the corrugation profile. The high precision was achieved by taking the ends of the $\mathrm{Al}$ mandrels (masked during electroforming) as reference points for finding the CWG axis (see Fig. 5). Straightening of the plated mandrels was performed after the completion of the EDM OD machining.

Before the final step of dissolving the aluminum, a highprecision cut at the ends of the trimmed mandrels was made
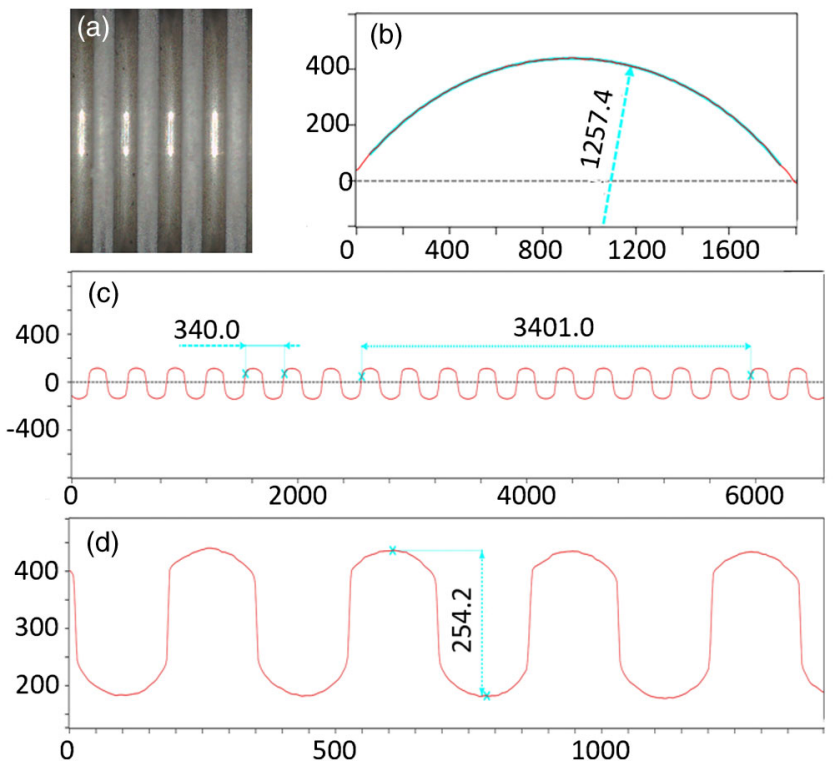

FIG. 4. (a) A fragment of the mandrel viewed under the microscope with a 150 times magnification. (b) Radius profile of the mandrel "tooth" that will form the CW "groove." (c) Longitudinal profile of the corrugation indicating uniform periodicity. (d) Corrugation detail indicating groove depth. All dimensions are in microns.

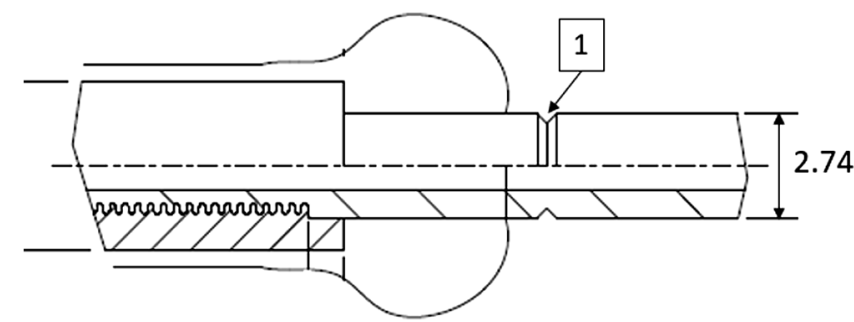

FIG. 5. Drawing of the end of the plated mandrel showing the diameter of the masked aluminum in millimeters and the location of the reference groove (1).

in the middle of the last corrugation tooth on both ends of the waveguide using the reference grooves machined at both ends of the Al mandrels (see Fig. 5).

Dimensional analysis was performed on two CWG samples which were cut in half longitudinally by wire EDM to expose the internal corrugations. The longitudinal profile measurements shown in Fig. 6(b) indicate excellent uniformity with the height of the corrugations, as well as with the periodicity. The average height of the corrugations is $249.7 \mu \mathrm{m}$, average periodicity is $340.7 \mu \mathrm{m}$, and deviations are within $\pm 5 \mu \mathrm{m}$. No cumulative error is evident across 10 periods. The cross-sectional profile indicates a uniform thickness of the deposited $\mathrm{Cu}$. The small "bumps" evident in the center of the profile are optical artifacts resulting from high reflectivity of the $\mathrm{Cu}$ surface. Figure 7 shows measurements of the internal corrugation radii for both the teeth and the grooves.

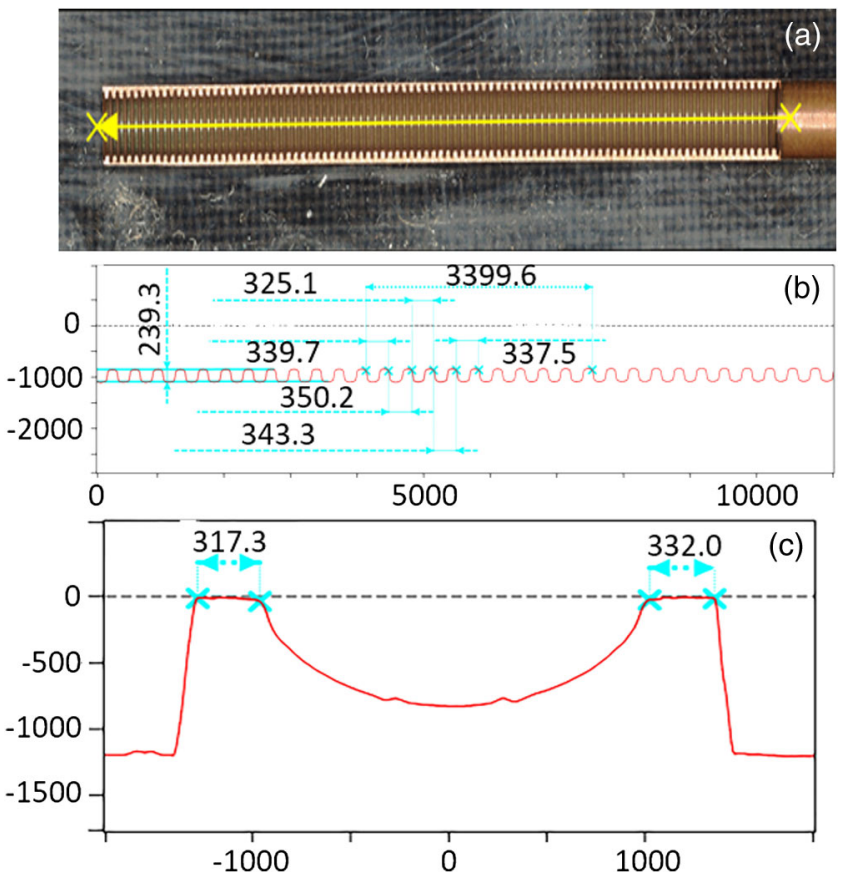

FIG. 6. (a) A fragment of the CWG with one half open. (b) Longitudinal profile of the corrugations measured along the line at the bottom of the CWG. (c) Cross-sectional profile measured in the middle of the tooth. All dimensions are in microns. 

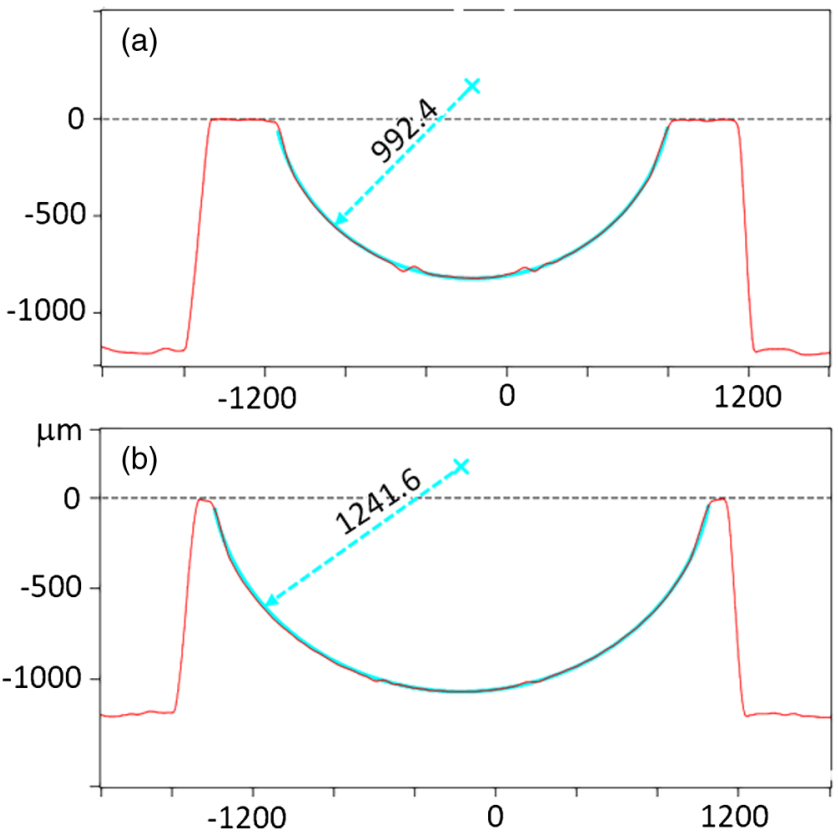

FIG. 7. The corrugation radii for (a) groove and for (b) tooth. All dimensions are in microns.

Surface roughness is difficult to quantify due to the compound curvature features, since the height differences are distorted by the curvature. However, a profile graph of diagonally cut images in Fig. 8(c) qualitatively shows the regularity of the corrugated geometry. The images in Figs. 8 (a) and 8(b) indicate a good surface roughness, although we plan further improvement using the hybrid electrochemical process. Small "bumps" seen next to the center are optical artifacts rather than actual surface distortions.
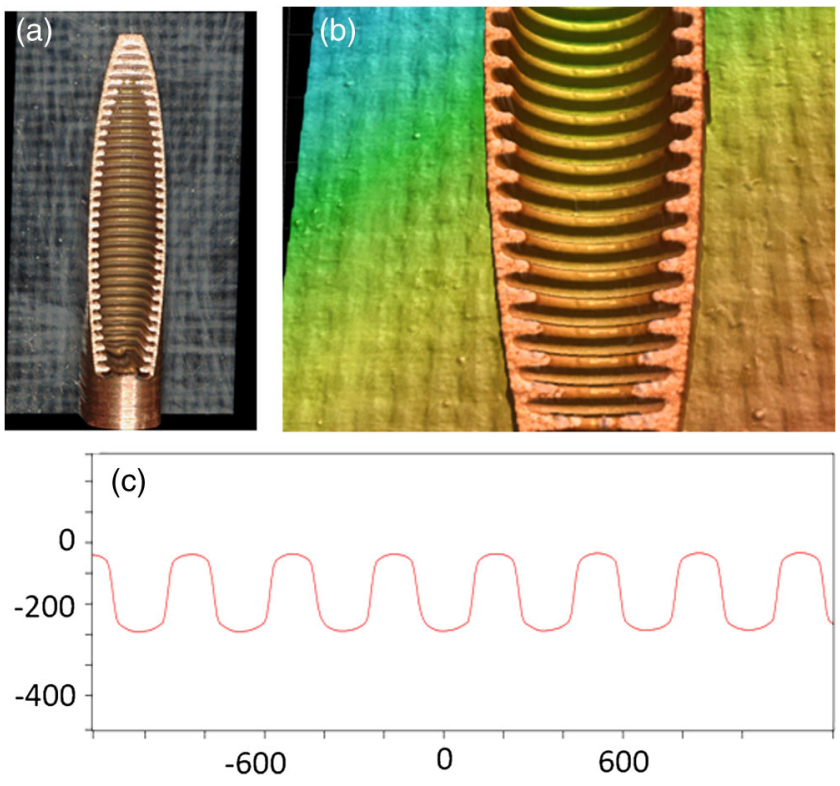

FIG. 8. Photographic images of (a) a diagonal cut CWG segment at 100 times magnification, (b) a 3D rendering of the diagonal cut segment, and (c) a profile graph of the corrugation. All dimensions are in microns.
TABLE I. Electron beam parameters at ATF.

\begin{tabular}{lccc}
\hline \hline Parameter & Value 1 & Value 2 & Unit \\
\hline Beam energy, $\mathcal{E}_{0}$ & 55 & 55 & $\mathrm{MeV}$ \\
Bunch charge, $Q$ & 150 & $130 / 170$ & $\mathrm{pC}$ \\
Charge distribution, $q(s)$ & flat top & flat top & \\
Bunch length, $\ell_{b} / c^{\mathrm{a}}$ & 1.5 & 5 & $\mathrm{ps}$ \\
Slice energy spread, $\sigma_{E}$ & tbd & 65 & $\mathrm{keV}$ \\
\hline \hline${ }^{\mathrm{a}} \mathrm{c}$ is the speed of light. & & &
\end{tabular}

\section{CORRUGATED WAVEGUIDE TESTING WITH THE ELECTRON BEAM}

We verified the compliance of the fabricated CWGs with the design in two sets of experiments performed at Brookhaven National Laboratory's Accelerator Test Facility (ATF) [22,23] using the electron beam with parameters listed in Table I. In both cases the bunch length $\ell_{b}$ of a flat-top charge distribution was controlled by the width of a vertical slit in a so-called energy collimator located inside of the dog-leg-type magnetic lattice at a place with a large dispersion function. The electron beam image at the energy collimator screen with the slit in the middle is shown in Fig. 9. By design, the energy collimator transmits electrons with the fixed central energy regardless of a shotto-shot energy jitter in the incoming electron bunch from the ATF's linear accelerator.

A schematic of the experimental setup is shown in Fig. 10. The electron bunch enters the experimental chamber and propagates through the CWG (1) where it produces sub-THz Čerenkov radiation. A $45^{\circ}$ end cut of the CWG is used for the efficient outcoupling of this radiation [24] which is captured by an off-axis parabolic mirror (2) and focused on a bolometer (8) after passing through a polymethylpentene (TPX) window (6) and a Michelson interferometer (7). The TPX window has close to $100 \%$ transmission efficiency for a sub-THz light.

The analysis of this setup performed using CST Microwave Studio [25] indicates that the transition radiation of the electron bunch at the exit of the CWG propagates forward and mostly misses the mirror, as seen in Fig. 11(a). Conversely, the sub-THz Čerenkov radiation produced inside the CWG exits at an angle to the electron

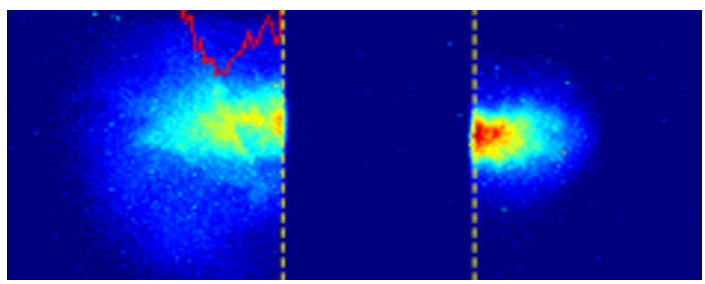

FIG. 9. The electron beam image at the energy collimator screen with a slit in the middle that cuts off bunch tails, transmits electrons only within a fixed energy range, and produces the electron bunch after the collimator with a predominantly flat-top charge distribution. 


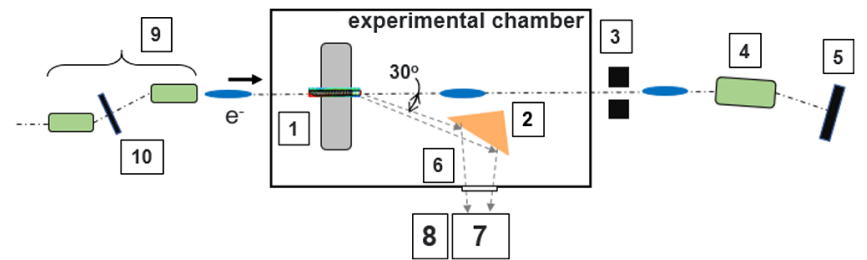

FIG. 10. A schematic of the experiment: (1) the device under test installed on the translation stage, (2) off-axis parabolic mirror, (3) collimator, (4) spectrometer magnet, (5) diagnostic screen, (6) TPX window, (7) Michelson interferometer, (8) bolometer, (9) a dog-leg lattice, (10) energy collimator.

bunch path. Diagrams in Figs. 11(b)-11(e) show an angular distribution in the horizontal plane of the monopole $\mathrm{TM}_{01}$, dipole $\mathrm{HEM}_{11}$, and quadrupole $\mathrm{HEM}_{21}$ modes of the sub$\mathrm{THz}$ Čerenkov radiation formed inside the CWG.

The photo in Fig. 12(a) shows a holder for CWGs mounted on the translation stage, a back side of the off-axis parabolic mirror, and the TPX window mounted on a bigger spool piece and illuminated by a green laser light. The photo in Fig. 12(b) shows a front view of the same holder and four CWGs. Their lengths from right to left are $L=96 \mathrm{~mm}$, $63 \mathrm{~mm}$, and $34 \mathrm{~mm}$. The fourth CWG seen on the photo was not used in the experiment. The second and third CWGs were obtained by cutting one full-length CWG into two unequal parts. One can also notice a green spot on the front side of the parabolic mirror that is left by the light coming from the green alignment laser and a red spot on the piece of paper temporarily placed next to the mirror's edge that is left by the light coming from the HeNe alignment laser. The red light was used for alignment of CWGs to the electron beam trajectory while the green light was used for alignment of the interferometer and the bolometer.

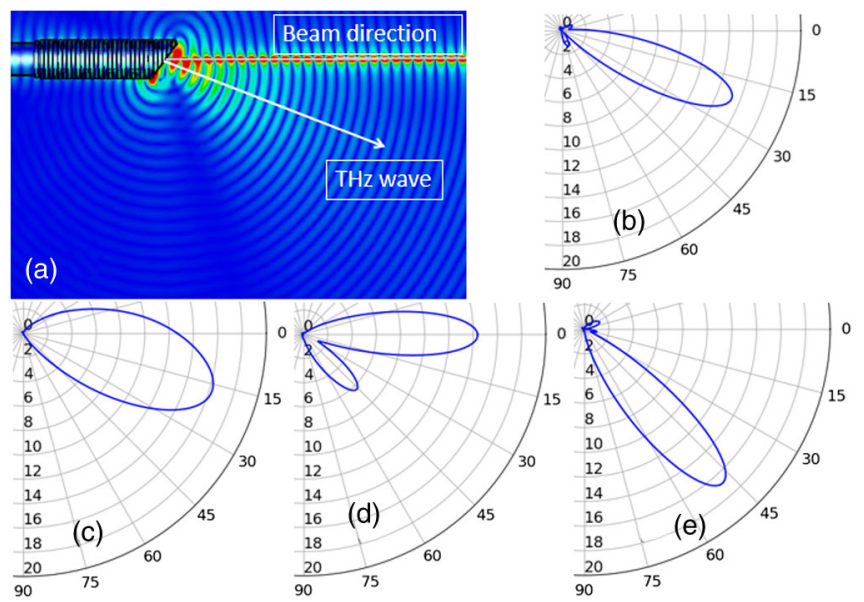

FIG. 11. (a) A snapshot of the radiation field calculated with CST Microwave Studio. The transition radiation is emitted forward. Panels (b-e) show diagrams of an angular distribution in the horizontal plane for the emission of the sub-THz Čerenkov radiation formed inside the $\mathrm{CWG}$ : (b) monopole mode, (c) dipole mode with vertical polarization, (d) dipole mode with horizontal polarization, and (e) quadrupole mode with vertical polarization.
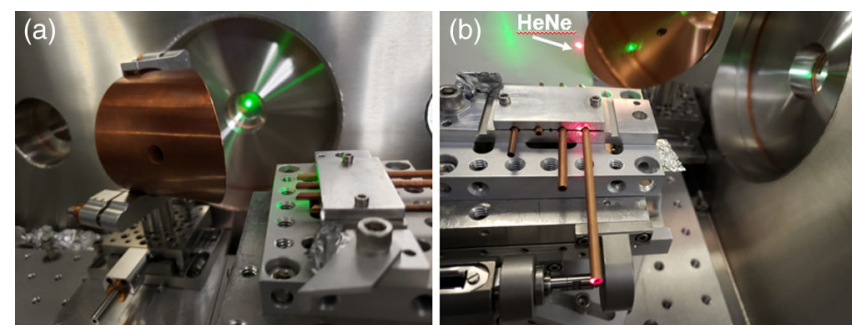

FIG. 12. A setup of the experiment with corrugated waveguides: (a) side view, (b) front view. See text for details.

In the first set of experiments we used the electron bunch with parameters from the first column in Table I and the interferometer to determine the frequencies of the monopole, dipole, and quadrupole modes of the sub- $\mathrm{THz}$ Čerenkov radiation produced by this bunch passing through the CWGs. Assuming a weak attenuation of the radiation in a relatively short CWG, and thus considering a constant amplitude of the radiation field as shown qualitatively in Fig. 13(a), we also

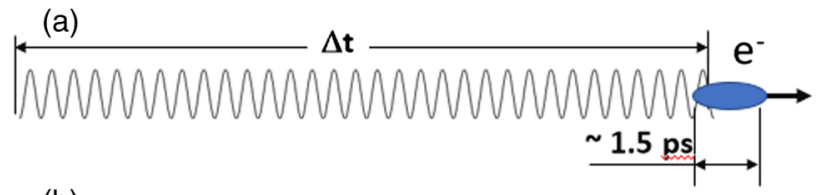

(b)

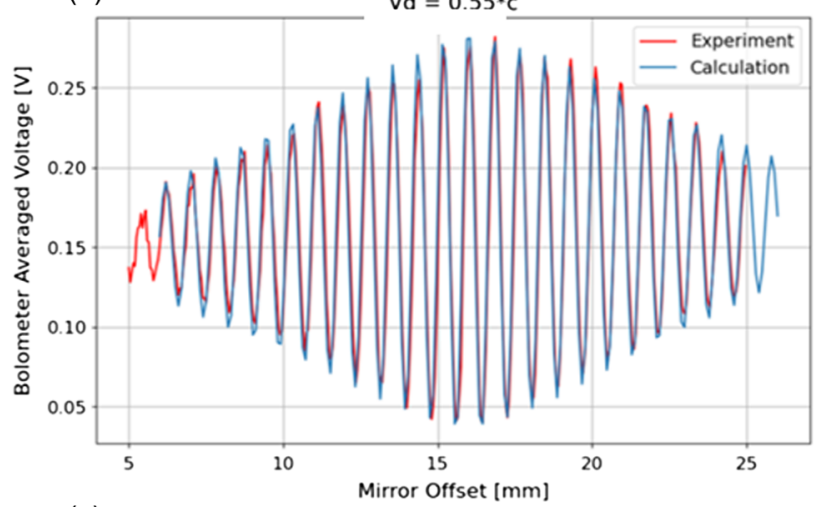

(c)

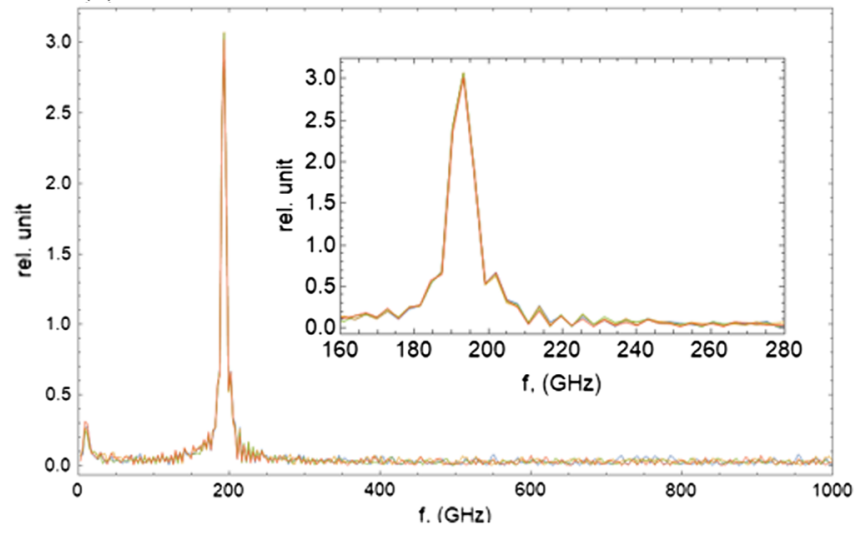

FIG. 13. (a) A qualitative illustration of the anticipated radiation. (b) The interferogram obtained when the electron bunch propagated through the CWG with the length $L=34 \mathrm{~mm}$. (c) The spectrum of the radiation emitted when the electron bunch propagated through the CWG with the length $L=96 \mathrm{~mm}$. 
measured the duration of the radiation pulse $\Delta t$ and obtained the group velocity $v_{g}$ with which the monopole mode propagates downstream of the CWG from the equation

$$
\Delta t=L\left(\frac{1}{v_{g}}-\frac{1}{v}\right)
$$

where $v=c \sqrt{1-1 / \gamma^{2}}$ is the electron bunch velocity and $\gamma$ is the relativistic factor. For that measurement we used the shortest CWG with $L=34 \mathrm{~mm}$ to be able to fit the entire interferogram within the available $24.5-\mathrm{mm}$ scanning range of the interferometer mirror, as seen in Fig. 13(b). Here we use the red color to show the measured interferogram and the blue color to show the best fit obtained using (i) the monopole mode frequency of $f_{M}=186 \mathrm{GHz}$ deduced from the Fourier transform of the interferogram, and (ii) $v_{g}=0.55 \mathrm{c}$ deduced from Eq. (1) while adjusting $\Delta t$ to fit the rising and falling interferogram "shoulders." The design values are $f_{M}=180 \mathrm{GHz}$ and $v_{g}=0.56 c$. The Fourier transform of the interferogram obtained using the second CWG with $L=$ $63 \mathrm{~mm}$ (not shown) gave the same frequency of $f_{M}=186 \pm 5 \mathrm{GHz}$. We also include in Fig. 13(c) the spectrum of the sub-THz radiation found by a Fourier transform of the interferogram obtained when the electron bunch propagated the longest corrugated waveguide with $L=96 \mathrm{~mm}$. The absence of high-order monopole modes in the spectrum validates one very important design goal for the corrugated waveguide. As seen in the inset, the frequency of the fundamental monopole mode is $192 \pm 5 \mathrm{GHz}$, which is within the expected fabrication error margin. The available scanning range of the interferometer defined the frequency resolution of $\simeq 10 \mathrm{GHz}$. Prior to this measurement we carefully adjusted the electron beam trajectory in the vertical and horizontal planes to the center of the CWG to minimize the dipole and quadrupole modes of Čerenkov radiation driven by the beam offset. However, to characterize these modes, we performed three measurements, sending the electron bunch through the longest CWG with horizontal offsets $\Delta x=0,200$, and $400 \mu \mathrm{m}$ using a precision translation stage from Newport Corporation, LTA series, with a $0.08 \mu \mathrm{m}$ minimum motion step. Figure 14 shows three spectra normalized at the $192-\mathrm{GHz}$ peak. According to Figs. 11(c) and 11(d), the second peak at $f_{D}=199 \mathrm{GHz}$ indicates the presence of a dipole $\mathrm{HEM}_{11}$ mode caused by the vertical beam offset. Calculations show that this offset increased by approximately $60 \mu \mathrm{m}$ between the first two measurements and the third measurement. The third peak at $f_{Q}=238 \mathrm{GHz}$ indicates the presence of a quadrupole $\mathrm{HEM}_{21}$ mode caused by the horizontal beam offset. The design values are $f_{D}=190 \mathrm{GHz}$ and $f_{Q}=230 \mathrm{GHz}$.

In the second set of experiments we used a very similar setup as in Fig. 10, but moved the off-axis parabolic mirror with the hole for the beam onto the beam path downstream of the CWG. The coherent backward diffraction radiation of the electron bunch entering this hole was used in the

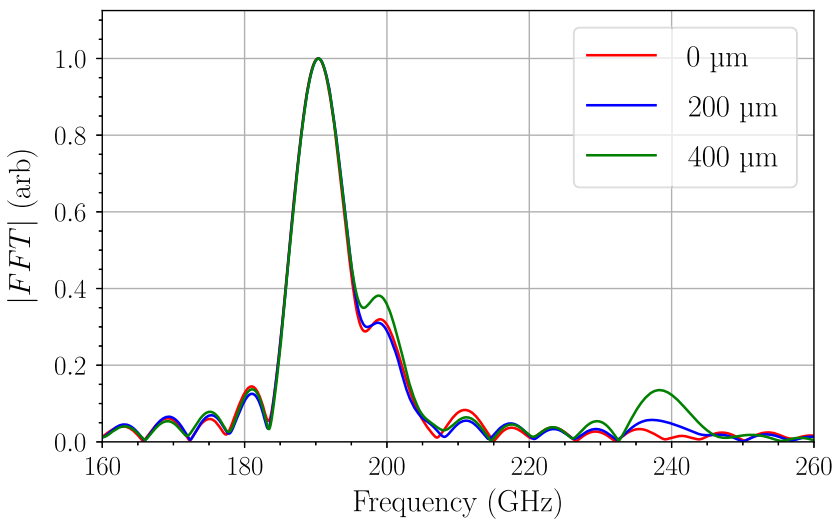

FIG. 14. Spectra normalized to the peak obtained during the horizontal translation sweep of the electron bunch trajectory.

interferometer for the bunch length measurements employing a similar technique to [26]. We also used a new set of CWGs with the lengths $L=74 \mathrm{~mm}, 93 \mathrm{~mm}$, and $100 \mathrm{~mm}$, although the profile of corrugations remained the same.

Since the corrugated waveguides have only one monopole mode with the frequency $f_{M} \simeq 180 \mathrm{GHz}$, we define the wakefield produced by the electron bunch as [27]

$E(s)=2 \kappa_{\|} \int_{0}^{s} \cos \left[k\left(s-s_{0}\right)\right] q\left(s_{0}\right) d s_{0}, \quad s \leq l_{b}$,

where $s$ is the electron coordinate inside the bunch with the head at $s=0, k=2 \pi f_{M} / c$ is the wave vector, $\kappa_{\|}=2 e / a^{2}$ is the loss factor in CGS units, and $q(s) \simeq$ $\frac{Q}{\ell_{b}}\left(\theta(s)-\theta\left(s-\ell_{b}\right)\right)$ is the charge distribution with a flattop profile, where $Q$ is the total bunch charge and $\theta(s)$ is the Heaviside step function. The bunch length in the experiment was controlled using the energy collimator. The wakefield acts on electrons propagating in the CWG and creates the energy modulation in the electron bunch that is particularly visible when the bunch length is comparable with the oscillation period $2 \pi / k$

$$
\Delta \mathcal{E}(s)=-2 \kappa_{\|} L \frac{Q}{k \ell_{b}} \sin (k s), s \leq l_{b}
$$

The goal of the experiment was to measure this energy modulation and to compare it with the calculations.

The experiment was carried out using the electron beam with parameters listed in the third column of Table I. In all measurements we employed the collimator (marked with the number (3) in a schematic of Fig. 10) to reduce the electron bunch charge after it already passed the CWG and minimize coherent synchrotron radiation in the spectrometer magnet (4). We calibrated the YAG diagnostic screen of the magnetic spectrometer (5) using the spectrometer magnet (4) and recorded the beam image shown in Fig. 15(d) after the electron bunch propagated a 

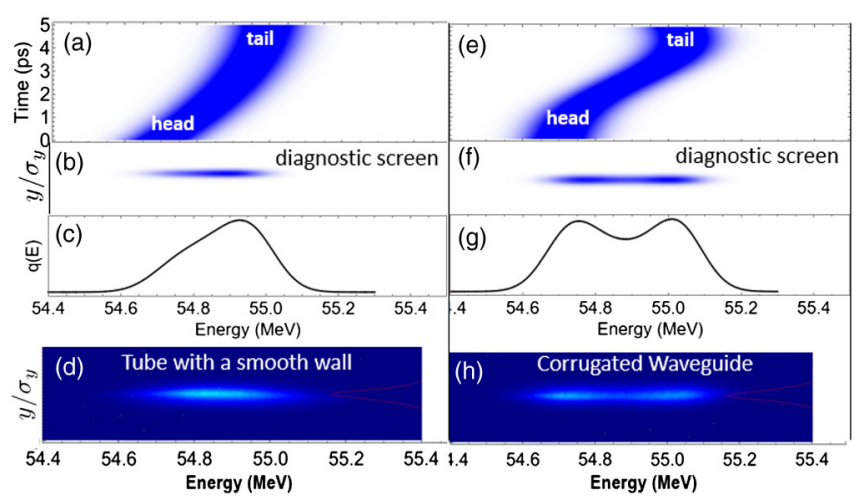

FIG. 15. Comparing measurements and simulations for the cases of the electron bunch propagating the tube with smooth wall (left column of plots) and the corrugated waveguide with $L=$ $74 \mathrm{~mm}$ (right column of plots). Plots (a) and (e) show the simulated distribution of electrons in the longitudinal phase space using energy on the horizontal axis and time on the vertical axis. Plots (b) and (f) show the simulated bunch image on the diagnostic screen using energy on the horizontal axis and the vertical coordinate $y$ normalized on the rms vertical size $\sigma_{y}$ on the vertical axis. Plots (c) and (g) show the simulated bunch energy distribution using energy on the horizontal axis and the charge in arbitrary units on the vertical axis. Plots (d) and (h) are similar to plots (b) and (f) and show the measured bunch image on the diagnostic screen using energy on the horizontal axis and the normalized vertical coordinate on the vertical axis.

102-mm-long tube without corrugations with a 2.1-mm ID. Exactly the same result was obtained when the electron bunch pass was unobstructed. In the other measurement, we ran the electron bunch in the linac on crest of the linac's radio frequency (rf) accelerating field and determined, though preliminary, the uncorrelated (slice) energy spread $\sigma_{E}$ using the beam image on the diagnostics screen. After that, we reproduced Fig. 15(d) in simulations, fitting the image by using as the main parameter the rf phase $\phi_{R F}$ for the electron bunch acceleration in the linac because it defines the energy chirp in the electron bunch, and also using $\sigma_{E}$ as the secondary parameter probing small deviations from the predetermined value. The result is shown in Fig. 15(b). The fitted values are $\sigma_{E}=65 \mathrm{keV}$ and $\phi_{R F}=6.0^{\circ}$. Using these values, we modeled the electron distribution in the longitudinal phase space and plotted it in Fig. 15(a). From that distribution we obtained the electron energy distribution and plotted it in Fig. 15(c).

After completing the above-described baseline measurements, we measured the impact of the wakefield on the electron bunch propagating the CWG. All three CWGs were used, and in Fig. 15 we present the measurement done using the CWG with $L=74 \mathrm{~mm}$. Two other measurements qualitatively were very similar and will be discussed later. Figure 15(h) shows an electron bunch image measured at the diagnostic screen. Using Eq. (3) and the abovedefined $\sigma_{E}$ and $\phi_{r f}$, we modeled the electron distribution in the longitudinal phase space [see Fig. 15(e)], the electron

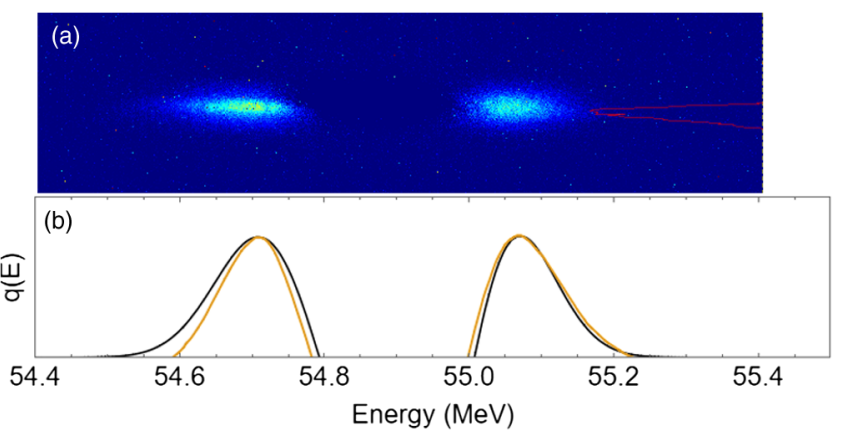

FIG. 16. (a) The difference between two diagnostics screen images, one taken when the electron bunch propagated the CWG with $L=74 \mathrm{~mm}$ and one taken when the electron bunch propagated the tube with the smooth wall. (b) The measured energy distribution of the electrons shown in the above plot (orange curve) and the calculated analogous energy distribution (black curve).

bunch image at the diagnostic screen [see Fig. 15(f)], and the electron energy distribution [see Fig. 15(g)]. The appearance of the two lobes on the sides that are absent in Fig. 15(d) clearly indicates the impact of the wakefield. Indeed, because the bunch length is comparable to the period of the wakefield oscillations of $5.6 \mathrm{ps,} \mathrm{observing}$ both decelerated and accelerated electrons is expected as confirmed by the results of the calculation shown in Figs. 15(e) and 15(g), and by the measurement in Fig. 15(h). We note that Fig. 15(f) closely resembles the measurement. A similar observation in the experiments with the corrugated waveguide was reported previously in [16].

For a more accurate comparison of the calculation and the measurement we numerically subtracted one experimental image in Fig. 15(d) from the other experimental image in Fig. 15(h) and replaced all negative values with zeros. By doing this, we highlighted locations of the side lobes plotted in Fig. 16(a) and obtained the energy distribution plotted in Fig. 16(b). After that we repeated the same analysis using calculated images in Figs. 15(b) and 15(e). The energy distributions are very close to each other. The distance between the peaks in the measurement is $0.36 \mathrm{MeV}$ and in the calculation is $0.36 \pm 0.04 \mathrm{MeV}$. The rms error was obtained considering $10 \%$ uncertainties in $Q, \sigma_{E}$, and $\phi_{r f}$, and was found to be mostly dominated by the uncertainty in $\phi_{r f}$.

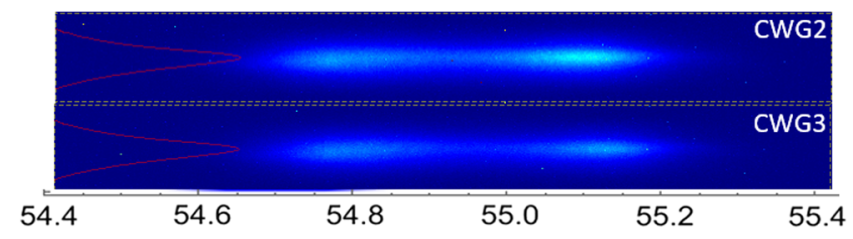

FIG. 17. The diagnostics screen images taken when the electron bunch propagated the CWG2 with $L=93 \mathrm{~mm}$ and the CWG3 with $L=100 \mathrm{~mm}$. 
The measurements performed using two other CWGs are presented in Fig. 17. The analysis of these measurements yielded very similar results.

\section{SUMMARY}

We developed a process for fabrication of a small size cylindrical corrugated waveguide, fabricated several samples, characterized them performing high-precision metrology, and tested with the electron beam at the ATF. In this test we verified the absence of the higher-order longitudinal modes in the wakefield, measured the frequency of the fundamental longitudinal wakefield mode, measured the group velocity of this mode, and measured the impact of the wakefield on the energy modulation of the electrons. All results agree with the calculations within the acceptable margin of fabrication errors. This work establishes the pass forward for the project aiming for the compact multiuser high repetition rate $\mathrm{x}$-ray free-electron laser facility envisioned in $[28,29]$. The technology described in the paper can be used for a fabrication of corrugated waveguides for an electron beam-based source producing with a high repetition rate ultra-short light pulses with sub-terahertz and terahertz carrier frequencies and hundreds of megawatt of a peak power envisioned in [16]. The corrugated structures discussed in the paper can also replace dielectric structures in the two-beam accelerator concept discussed in [30].

\section{ACKNOWLEDGMENTS}

This manuscript is based upon work supported by Laboratory Directed Research and Development (LDRD) funding from Argonne National Laboratory, provided by the Director, Office of Science, of the U.S. Department of Energy under Contract No. DE-AC02-06CH11357. Useful discussion with S. Doran, G. Navrotski, B. Popovic, J. Power, and J Xu of A. N. L. are gratefully acknowledged. We thank the Accelerator Test Facility staff at BNL for support during the experiment and M. Polyanskiy of BNL for support in using easyFG software for image processing.

[1] G. Voss and T. Weiland, The wake field acceleration mechanism, DESY Technical Report No. DESY-82-074, 1982.

[2] R. J. Briggs, T. J. Fessenden, and V. K. Neil, Electron autoacceleration, in Proceedings, 9th International Conference on the High-Energy Accelerators (SLAC-R839, 1974), p. 278.

[3] M. Friedman, Autoacceleration of an Intense Relativistic Electron Beam, Phys. Rev. Lett. 31, 1107 (1973).

[4] E. A. Perevedentsev and A. N. Skrinsky, On the use of the intense beams of large proton accelerators to excite the accelerating structure of a linear accelerator, in Proceedings of 6th All-Union Conference Charged Particle Accelerators, Dubna (Institute of Nuclear Physics, Novosibirsk, USSR, 1978), Vol. 2, p. 272; , English version is available in Proceedings of the 2nd ICFA Workshop on Possibilities and Limitations of Accelerators and Detectors (CERN, Geneva, Switzerland, 1980), p. 61.

[5] Y. Chin, The wake field acceleration using a cavity of elliptical cross section, in 12th International Linear Accelerator Conference (GSI, Darmstadt, Germany, 1984), pp. 159-161.

[6] W. Gai, P. Schoessow, B. Cole, R. Konecny, J. Norem, J. Rosenzweig, and J. Simpson, Experimental Demonstration of Wake-field Effects in Dielectric Structures, Phys. Rev. Lett. 61, 2756 (1988).

[7] W. H. Tan, P. Piot, and A. Zholents, Formation of temporally shaped electron bunches for beam-driven collinear wakefield accelerators, Phys. Rev. Accel. Beams 24, 051303 (2021).

[8] K. Bane and G. Stupakov, Terahertz radiation from a pipe with small corrugations, Nucl. Instrum. Methods Phys. Res., Sect. A 677, 67 (2012).

[9] A. Novokhatski, Wakefield potentials of corrugated structures, Phys. Rev. ST Accel. Beams 18, 104402 (2015).

[10] K. Bane, G. Stupakov, and I. Zagorodnov, Analytical formulas for short bunch wakes in a flat dechirper, Phys. Rev. Accel. Beams 19, 084401 (2016).

[11] P. Emma, M. Venturini, K. L. F. Bane, G. Stupakov, H.-S. Kang, M. S. Chae, J. Hong, C.-K. Min, H. Yang, T. Ha, W. W. Lee, C. D. Park, S. J. Park, and I. S. Ko, Experimental Demonstration of Energy-Chirp Control in Relativistic Electron Bunches Using a Corrugated Pipe, Phys. Rev. Lett. 112, 034801 (2014).

[12] Z. Zhang, K. Bane, Y. Ding, Z. Huang, R. Iverson, T. Maxwell, G. Stupakov, and L. Wang, Electron beam energy chirp control with a rectangular corrugated structure at the linac coherent light source, Phys. Rev. ST Accel. Beams 18, 010702 (2015).

[13] H. Deng et al., Experimental Demonstration of Longitudinal Beam Phase-Space Linearizer in a Free-Electron Laser Facility by Corrugated Structures, Phys. Rev. Lett. 113, 254802 (2014).

[14] S. Bettoni, P. Craievich, A. A. Lutman, and M. Pedrozzi, Temporal profile measurements of relativistic electron bunch based on wakefield generation, Phys. Rev. Accel. Beams 19, 021304 (2016).

[15] G. Stupakov, Using pipe with corrugated walls for a subterahertz free electron laser, Phys. Rev. ST Accel. Beams 18, 030709 (2015).

[16] K. Bane, G. Stupakov, S. Antipov, M. Fedurin, K. Kusche, C. Swinson, and D. Xiang, Measurements of terahertz radiation generated using a metallic, corrugated pipe, Nucl. Instrum. Methods Phys. Res., Sect. A 844, 121 (2017).

[17] A. E. Siy, G. J. Waldschmidt, and A. A. Zholents, Design of a compact wakefield accelerator based on a corrugated waveguide, in Proceedings of 2019 North American Particle Accelerator Conference (NAPAC2019), Lansing, MI, USA, September 1-6, 2019 ( JACoW, Geneva, Switzerland, 2019), pp. 232-235.

[18] K. Suthar et al., Investigation of various fabrication methods to produce a $180 \mathrm{GHz}$ corrugated waveguide structure in $2 \mathrm{~mm}$-diameter $-0.5 \mathrm{~m}$-long copper tube for the compact wakefield accelerator for FEL facility, in Proceedings of 2019 North American Particle Accelerator 
Conference (NAPAC2019), Lansing, MI, USA, September 1-6, 2019 (JACoW, Geneva, 2019), pp. 286-289.

[19] W. Jansma, S. Sorsher, K. Suthar, E. Trakhtenberg, and A. Zholents, Metrology in the R\&D for the high repetition rate multi-user x-ray free-electron laser, J. CMSC (Autumn 2019), https://www.cmsc.org/cmsc-2019-presentation--metrology-in-the-rd-for-a-high-repetition-rate-multi-userX-ray-free-electron-laser-facility.

[20] J. W. Dini and D. D. Snyder, Electrodeposition of copper, in Modern Electroplating (John Wiley and Sons, New York, 2010), Chap. 2, pp. 33-78, https://onlinelibrary .wiley.com/doi/pdf/10.1002/9780470602638.ch2.

[21] P. Fallah, L. A. Hof, and R. Wuthrich, Fabrication of highthickness and low surface roughness metal parts by a hybrid electrochemical manufacturing process, Adv. Indust. Manufact. Eng. 2, 100034 (2021).

[22] https://www.bnl.gov/atf/.

[23] V. Yakimenko, The accelerator test facility at Brookhaven: Main capabilities, AIP Conf. Proc. 737, 677 (2004), https://aip.scitation.org/doi/pdf/10.1063/1 .1842608 .

[24] S. Antipov, S. V. Baryshev, R. Kostin, S. Baturin, J. Qiu, C. Jing, C. Swinson, M. Fedurin, and D. Wang, Efficient extraction of high power $\mathrm{THz}$ radiation generated by an ultra-relativistic electron beam in a dielectric loaded waveguide, Appl. Phys. Lett. 109, 142901 (2016).
[25] CST Microwave Studio, Dassault Systems, Inc. (2020), https://www.3ds.com/products-services/simulia/products/ cst-studio-suite/.

[26] P. Muggli, V. Yakimenko, M. Babzien, E. Kallos, and K. P. Kusche, Generation of Trains of Electron Microbunches with Adjustable Subpicosecond Spacing, Phys. Rev. Lett. 101, 054801 (2008).

[27] A. Chao, K. H. Mess, M. Tigner, and F. Zimmermann, Handbook of Accelerator Physics and Engineering, 2nd ed. (World Scientific, Singapore, 2013).

[28] A. Zholents et al., A conceptual design of a compact wakefield accelerator for a high repetition rate multi user $\mathrm{X}$-ray free electron laser facility, in Proceedings of 9th International Particle Accelerator Conference (IPAC'18), Vancouver, BC, Canada, April 29-May 4, 2018, International Particle Accelerator Conference No. 9 ( JACoW Publishing, Geneva, Switzerland, 2018), pp. 1266-1268, 10.18429/JACoW-IPAC2018-TUPMF010.

[29] A. Zholents, S. Baturin, D. Doran, W. Jansma, M. Kasa, A. Nassiri, P. Piot, J. Power, A. Siy, S. Sorsher, K. Suthar, W. Tan, E. Trakhtenberg, G. Waldschmidt, and J. Xu, A compact high repetition rate free-electron laser based on the advanced wakefield accelerator technology, https://ipac2020.vrws.de/ html/author.htm (2020), presented at IPAC'20 in Caen, France.

[30] C. Jing, Dielectric wakefield accelerators, Rev. Accel. Sci. Techol. 09, 127 (2016). 